\title{
Norois
}

Environnement, aménagement, société

La Loire. Sociétés, risques, paysages, environnement

\section{L'assèchement de cours d'eau dans le bassin de la Maine entre 1989 et 1992}

The drying up of streams in the Maine basin from 1989 to 1992

Jean-Pierre Larue et Alain Giret

\section{(2) OpenEdition}

1 Journals

\section{Édition électronique}

URL : https://journals.openedition.org/norois/944

DOI : $10.4000 /$ norois. 944

ISBN : 978-2-7535-1540-6

ISSN : $1760-8546$

\section{Éditeur}

Presses universitaires de Rennes

Édition imprimée

Date de publication : 1 septembre 2004

Pagination : 117-133

ISBN : 978-2-7535-0054-9

ISSN : 0029-182X

\section{Référence électronique}

Jean-Pierre Larue et Alain Giret, « L'assèchement de cours d'eau dans le bassin de la Maine entre 1989 et 1992 », Norois [En ligne], 192 | 2004/3, mis en ligne le 24 septembre 2008, consulté le 13 janvier 2022. URL : http://journals.openedition.org/norois/944 ; DOI : https://doi.org/10.4000/norois. 944

Ce document a été généré automatiquement le 13 janvier 2022.

(c) Tous droits réservés 


\section{L'assèchement de cours d'eau dans le bassin de la Maine entre 1989 et} 1992

The drying up of streams in the Maine basin from 1989 to 1992

Jean-Pierre Larue et Alain Giret

\section{NOTE DE L'ÉDITEUR}

Cet article a été reçu en août 1999, repris par la revue « nouvelle formule » en novembre 2003 et définitivement accepté le $1^{\text {er }}$ juin 2004.

Nous remercions les organismes qui nous ont aimablement communiqué leurs données hydrologiques et météorologiques, et tout particulièrement M. Vidal, de la DIREN, ${ }^{\text {me }}{ }^{\text {Maupas, }}$ de l'agence de bassin Loire-Bretagne, et M. Portela, de la station météorologique d'Arnage, $M$. Marandola (Paris 12) pour la mise au net des figures ainsi que les deux relecteurs anonymes qui ont contribué à l'amélioration du manuscrit. 
Tableau 1 : Caractéristiques hydrologiques des cours d'eau asséchés en 1976, 1990, 1991 et 1992 Hydrological features of dried up streams in 1976, 1990, 1991 and 1992

\begin{tabular}{|c|c|c|c|c|c|c|}
\hline & BV & Rhonne & $\begin{array}{l}\text { Oudon } \\
\text { (Marcillé) }\end{array}$ & Cheran & Uzure & Vaige \\
\hline & $\mathrm{S}(\mathrm{km})$ & 77 & 734 & 85 & 21 & 233 \\
\hline moy $61-88$ & \begin{tabular}{|l}
$P(m m)$ \\
$E c(m m)$ \\
$Q$ spe \\
min m \\
min j
\end{tabular} & 728 & $\begin{array}{l}709 \\
166 \\
5,27 \\
0,95 \\
0,14 \\
\end{array}$ & \begin{tabular}{|l|}
667 \\
186 \\
5,55 \\
0,4 \\
0,25 \\
\end{tabular} & \begin{tabular}{|l|}
714 \\
174 \\
8,85 \\
0,95 \\
0,66 \\
\end{tabular} & $\begin{array}{l}732 \\
210 \\
7,55 \\
0,66 \\
0,21 \\
\end{array}$ \\
\hline 1976 & \begin{tabular}{|l}
$P(m m)$ \\
$E c(m m)$ \\
min $m$ \\
$D A$ \\
$D E$ \\
$D E$
\end{tabular} & 497 & \begin{tabular}{|l|}
535 \\
35 \\
0 \\
212 \\
91 \\
\end{tabular} & \begin{tabular}{|l|}
555 \\
76 \\
0,012 \\
181 \\
36 \\
\end{tabular} & & \\
\hline 1989 & \begin{tabular}{|l}
$P(m m)$ \\
$E c(m m)$ \\
$Q s p e$ \\
$m i n m$ \\
$m i n j$ \\
$D E$ \\
$D E S$ \\
$D A$ \\
\end{tabular} & \begin{tabular}{|l|}
598 \\
69 \\
2,17 \\
0,18 \\
0,007 \\
69 \\
35 \\
0 \\
\end{tabular} & \begin{tabular}{|l|l|}
630 \\
100 \\
3,16 \\
0,023 \\
0,004 \\
138 \\
115 \\
0 \\
\end{tabular} & \begin{tabular}{|l|}
580 \\
135 \\
4,28 \\
0,27 \\
0,11 \\
81 \\
12 \\
0 \\
\end{tabular} & \begin{tabular}{|l|}
597 \\
118 \\
3,71 \\
0,47 \\
0,14 \\
32 \\
3 \\
0 \\
\end{tabular} & \begin{tabular}{|l|}
6500 \\
123 \\
3,91 \\
0,064 \\
0,03 \\
128 \\
95 \\
0 \\
\end{tabular} \\
\hline 1990 & \begin{tabular}{|l}
$\mathrm{P}(\mathrm{mm})$ \\
$\mathrm{Ec}(\mathrm{mm})$ \\
$Q$ spe \\
min $\mathrm{m}$ \\
$\mathrm{DE}$ \\
$\mathrm{DES}$ \\
$\mathrm{DA}$ \\
\end{tabular} & \begin{tabular}{|l|}
609 \\
63 \\
1,97 \\
0 \\
71 \\
66 \\
53 \\
\end{tabular} & \begin{tabular}{|l|}
591 \\
90 \\
2,86 \\
0 \\
166 \\
134 \\
47 \\
\end{tabular} & \begin{tabular}{|l|}
540 \\
136 \\
4,31 \\
0 \\
103 \\
60 \\
41 \\
\end{tabular} & \begin{tabular}{|l}
577 \\
126 \\
4 \\
0 \\
110 \\
78 \\
31 \\
\end{tabular} & \begin{tabular}{|l|}
626 \\
107 \\
3,41 \\
0 \\
107 \\
96 \\
51 \\
\end{tabular} \\
\hline 1991 & \begin{tabular}{|l}
$\mathrm{P}(\mathrm{mm})$ \\
$\mathrm{Ec}(\mathrm{mm})$ \\
$Q \mathrm{spe}$ \\
$\mathrm{min} \mathrm{m}$ \\
$\mathrm{DE}$ \\
$\mathrm{DES}$ \\
$\mathrm{DA}$ \\
\end{tabular} & \begin{tabular}{|l|}
555 \\
75 \\
2,38 \\
0 \\
76 \\
75 \\
73 \\
\end{tabular} & \begin{tabular}{|l|}
581 \\
80 \\
2,52 \\
0,038 \\
138 \\
109 \\
14 \\
\end{tabular} & \begin{tabular}{|l|}
555 \\
127 \\
4,02 \\
0,09 \\
137 \\
64 \\
15 \\
\end{tabular} & \begin{tabular}{|l|}
559 \\
104 \\
3,29 \\
0,047 \\
129 \\
60 \\
28 \\
\end{tabular} & \begin{tabular}{|l|}
617 \\
122 \\
3,86 \\
0,004 \\
101 \\
73 \\
26 \\
\end{tabular} \\
\hline 1992 & \begin{tabular}{|l}
$\mathrm{P}(\mathrm{mm})$ \\
$\mathrm{Ec}(\mathrm{mm})$ \\
$\mathrm{Q}$ spe \\
min m \\
$\mathrm{DE}$ \\
$\mathrm{DES}$ \\
$\mathrm{DA}$ \\
\end{tabular} & \begin{tabular}{|l|}
621 \\
35 \\
1,09 \\
0,05 \\
1,23 \\
78 \\
15 \\
\end{tabular} & \begin{tabular}{|l|}
658 \\
43 \\
1,35 \\
0,026 \\
281 \\
213 \\
14 \\
\end{tabular} & \begin{tabular}{|l|}
615 \\
85 \\
2,68 \\
0,176 \\
172 \\
65 \\
11 \\
\end{tabular} & $\begin{array}{l}618 \\
42 \\
1,33 \\
0 \\
153 \\
143 \\
105 \\
\end{array}$ & \begin{tabular}{|l|}
634 \\
74 \\
2,34 \\
0 \\
160 \\
117 \\
52 \\
\end{tabular} \\
\hline
\end{tabular}

BV : bassin versant ; : surface ; $P$ : précipitations ; Ec : lame d'eau écoulée ; Qspe : module spécifique $\left(\mathrm{l} / \mathrm{s} / \mathrm{km}^{2}\right) ;$ min $\mathrm{m}$ : minimum mensuel $\left(1 / \mathrm{s} / \mathrm{km}^{2}\right) ; \mathrm{DE}$ : durée des étiages en jours ; DES : durée des étiages sévères (< $1 / 2$ débit limite d'étiage) en jours ; DA : durée d'assèchement en jours BV: catchment area; S: surface; P: rainfalls; E: flow; Qspe: specific module (I/s/km2); min m: monthly minimum (I/s/km2); DE: duration in days of lowest rate of flow; DES: duration of worst lowest rate of flow (< 1/2 limit rate of flow at its lowest) in days; DA: duration of drying up in days

1 Situé aux confins du Bassin parisien et du Massif armoricain, le bassin de la Maine (22 $185 \mathrm{~km}^{2}$ ) appartient aux régions de climat océanique qui ont été sévèrement touchées par les sécheresses entre 1989 et 1992. Caractérisés par des régimes pluvio-évaporaux, les cours d'eau, soumis à 34 mois de déficit pluviométrique durant les quatre années hydrologiques (d'août 1988 à septembre 1992), ont subi des étiages plus ou moins graves qui se différencient des basses eaux ordinaires de saison chaude par leur nature exceptionnelle. Cependant, seul un petit nombre de bassins versants (fig. 1 et tableau 1) ont atteint à plusieurs reprises le stade ultime d'assèchement des lits mineurs. Mérillon (1992) estime, pour la France entière, que $11000 \mathrm{~km}$ de rivières ont été asséchées à la fin des étés 1989 et 1990. Ces cas d'assèchement, qui restent exceptionnels dans le bassin de la Maine, sont localisés pour la plupart sur le socle du Massif armoricain et affectent des cours d'eau comme la Vaige, l'Oudon et ses tributaires (l'Uzure et le Chéran) mais on en rencontre également sur la couverture sédimentaire du Bassin parisien comme le Rhonne. Aussi méritent-ils une analyse détaillée afin de mieux comprendre le fonctionnement hydrologique de leurs bassins versants. 
Figure 1 : Localisation et structure Location and structure

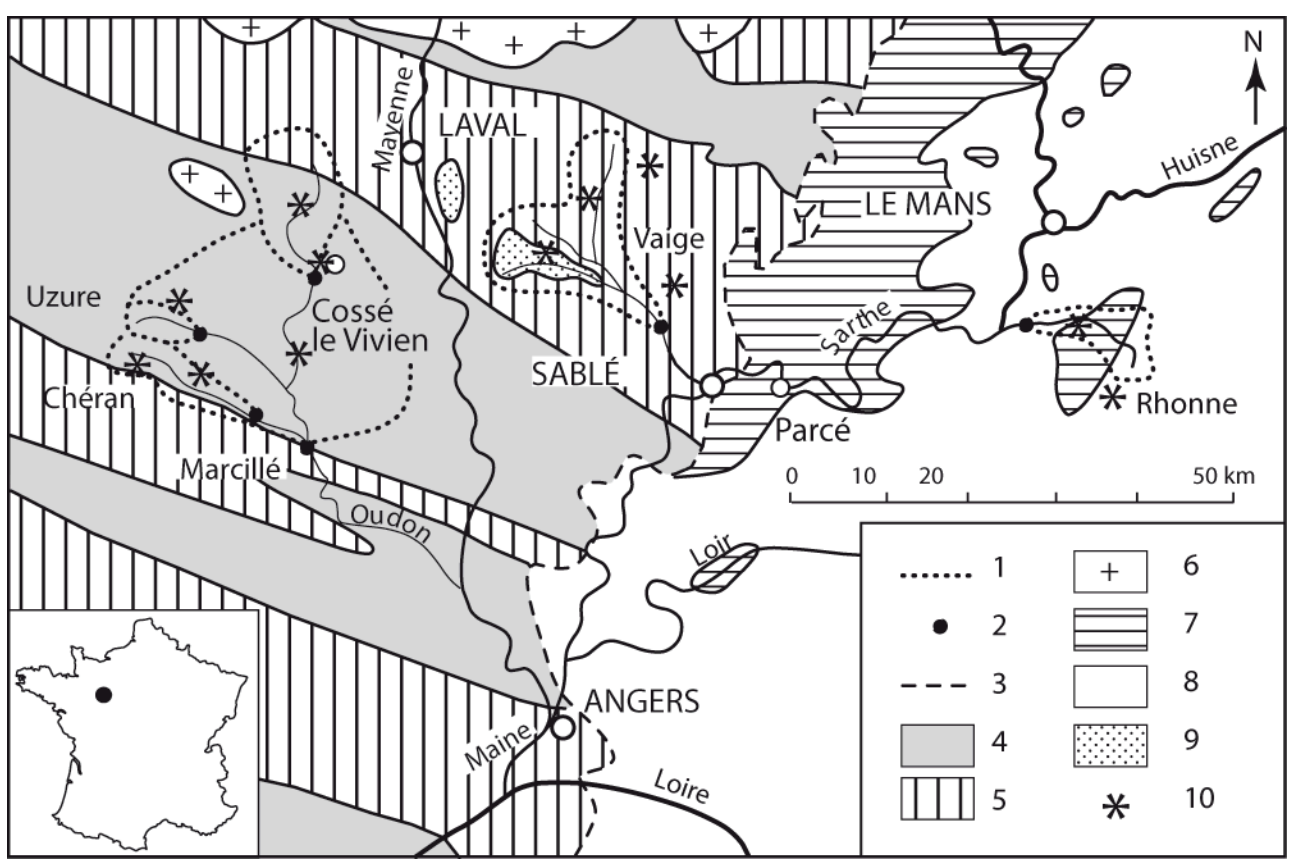

1 : limite des bassins versants ; 2 : limnigraphe ; 3 : limite socle (à l'ouest)-couverture sédimentaire (à l'est) ; 4 : schistes briovériens, anticlinal ; 5 : synclinal primaire, schistes, grés, calcaires ; 6 : batholite granitique ; 7 : Jurassique argilo-marneux ; 8 : Crétacé sableux et crayeux ; 9 : dépôts détritiques pliocènes peu épais ; 10 : poste pluviométrique.

1: limit of catchment areas; 2: limnigraph; 3 : limit basement (to the W)-sediment cover (to the E); 4: Brioverian shale, anticline; 5: primary syncline, shale, sandstone, limestone ; 6: granite batholith; 7: Jurassic marly clay; 8: sandy and chalky Cretaceous; 9: thin Pliocene detrital deposit; 10: pluviometrical station.

2 Nous avons choisi les bassins cités précédemment car ils disposent de stations hydrométriques gérées par la DIREN Pays de la Loire et de relevés pluviométriques assurés par la Météorologie Nationale (fig. 1). Ces données journalières permettent de suivre l'évolution des bilans hydriques de chaque bassin et de construire des hydrogrammes qui, décomposés selon la méthode de Butler (1957) utilisée par Lambert (1975), Cosandey (1993 et 2003), Giret (1993, 1999 et 2002), révèlent la part de l'écoulement rapide de crue ou ERC (expression plus appropriée, pour définir la part des pluies atteignant rapidement les chenaux, que les anciens termes de ruissellement superficiel et d'écoulement hypodermique, qu'il est très difficile de séparer quand on ne dispose que des valeurs moyennes quotidiennes de l'écoulement) et de l'écoulement de base. 
Figure 2 : Dates d'assèchement de l'Oudon et ses tributaires, de la Vaige et du Rhonne Dates of drying up of the Oudon and its tributaries, the Vaige and the Rhonne

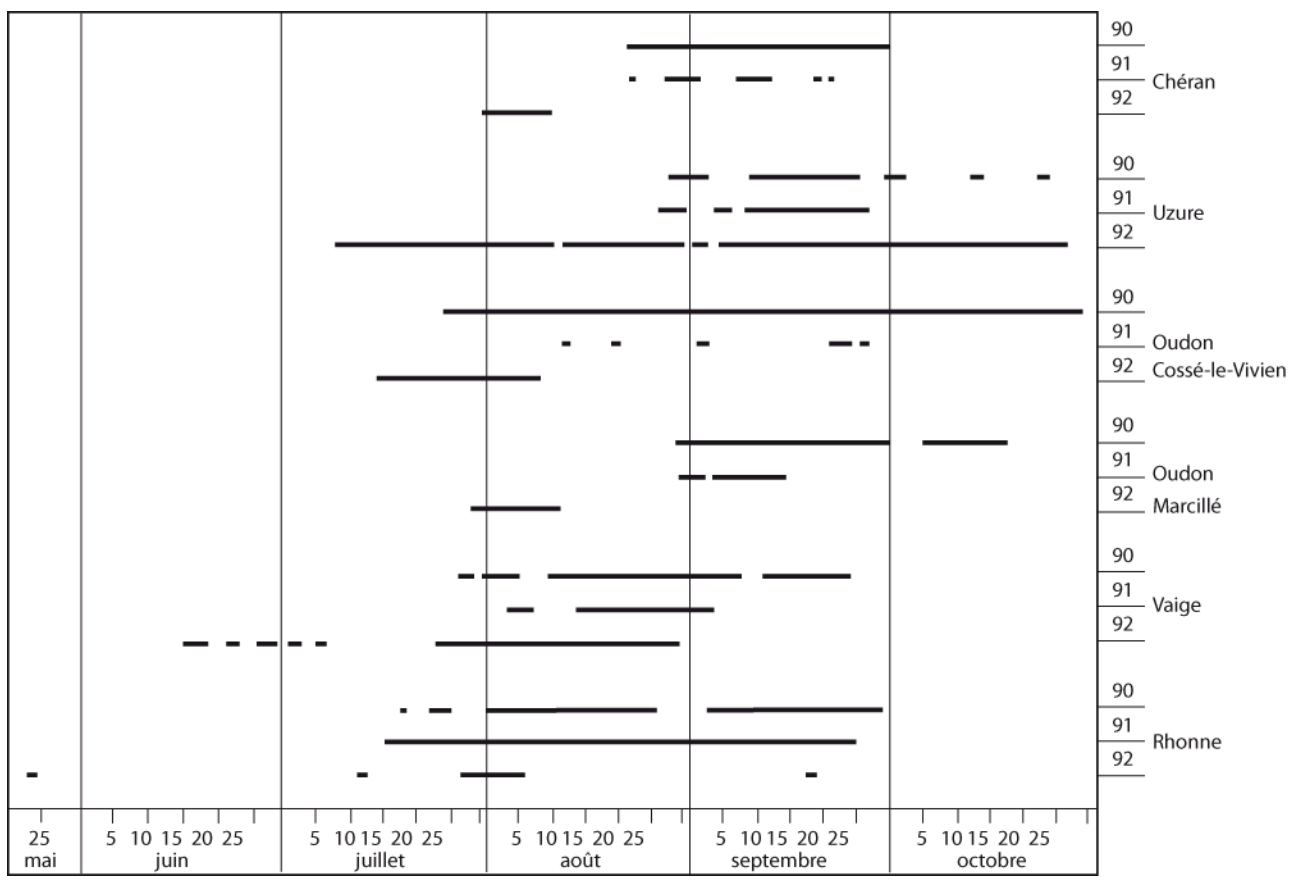

3 Comme les conditions climatiques et topographiques apparaissent relativement homogènes pour l'ensemble du bassin de la Maine, cette étude souligne le rôle prédominant des facteurs morphostructuraux et des formations superficielles sur l'écoulement, sans toutefois négliger celui des ripisylves et des activités humaines.

\section{Chronologie des assèchements}

4 La figure 2 montre l'évolution de la durée d'assèchement entre 1989 et 1992 pour les bassins disposant de données pluviométriques et hydrométriques. Inconnu en 1989, l'assec dure entre 11 jours pour le Chéran en 1992 et 105 jours pour l'Uzure en 1992, sans qu'il ne soit possible d'établir un classement par année ou par bassin: la plus longue période d'assèchement est enregistrée en 1992 pour l'Uzure (105 jours) et la Vaige ( 52 jours), en 1990 pour le Rhonne (53 jours) et l'Oudon ( 47 jours) et en 1991 pour le Rhonne (73 jours). L'assec concerne tout le linéaire des cours d'eau dans les petits bassins versants du Massif armoricain (Uzure et Chéran) mais exclut le secteur le plus en aval, pour la Vaige et l'Oudon, et le cours amont pour le Rhonne.

5 L'assec se produit principalement durant la saison estivale, mais il peut empiéter plus ou moins largement sur l'automne et le printemps. Par exemple, il débute le 23 mai sur le Rhonne en 1992, mais seulement le 30 août sur l'Oudon en 1991 et se termine le 29 octobre sur l'Oudon à Cossé-le-Vivien en 1990 mais dès le 8 août en 1992 pour la même station. Il comprend une seule phase pour le Rhonne en 1991, pour l'Oudon en 1992 et pour le Chéran en 1990 et 1992 mais on relève jusqu'à six périodes séparées par de l'écoulement pour la Vaige en 1992.

6 La construction des courbes de débits classés, comme par exemple pour l'Oudon à Marcillé (fig. 3), révèle que les années de tarissement dessinent des courbes plus accidentées que les autres qui se rapprochent de la droite en échelle logarithmique. 
L'année 1992 est caractérisée par le plus grand nombre de jours ayant un faible débit : le débit caractéristique dépassé 180 jours (DC 6) n'atteint que $0,27 \mathrm{l} / \mathrm{s} / \mathrm{km}^{2}$, contre 1,18 en $1989,0,67$ en 1990 et 1,27 en 1991. Les autres cours d'eau fournissent des données semblables.

Figure 3 : Débits classés de l'Oudon de 1988 à 1992

Recorded discharge of the Oudon from 1988 to 1992

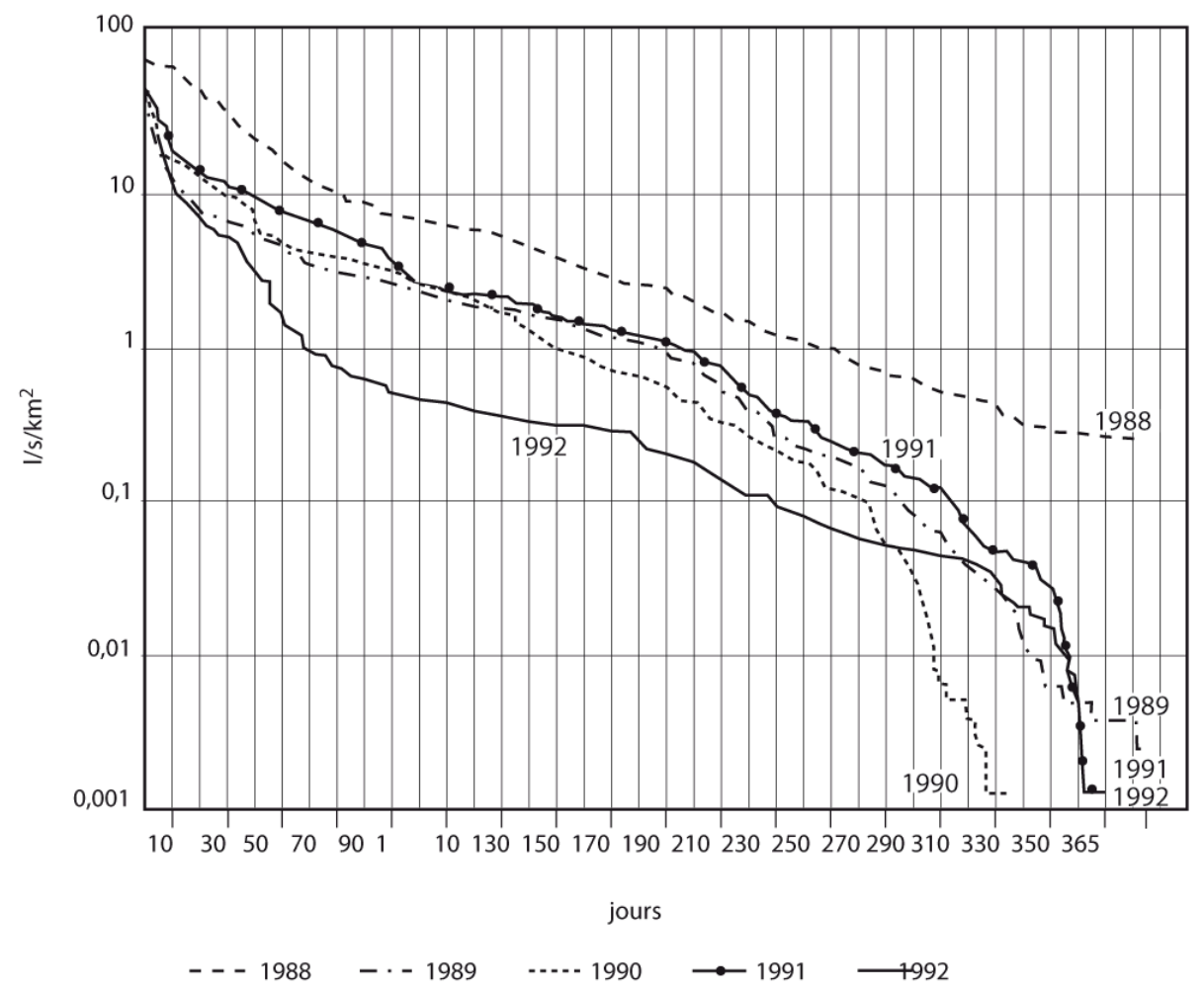

\section{L'enseignement des hydrogrammes}

7 La Vaige, le Rhonne et l'Uzure sont équipés de limnigraphes à flotteur et les mesures effectuées sur déversoir fournissent des données relativement précises même pour de très faibles débits, toutefois, en dessous d'un certain seuil que l'on peut évaluer entre 3 et $5 \mathrm{l} / \mathrm{s}$, les enregistrements peuvent manquer de fiabilité. Les hydrogrammes, construits en périodes d'étiage, ont été décomposés de façon à mettre en lumière les phases d'assèchement. La méthode purement analytique repère les points de rebroussement des vecteurs, points auxquels on attribue une lettre. Le tarissement de l'écoulement se traduit par une ligne brisée, par exemple ABCD sur la Vaige en 1990. La figure 4 illustre les variations des débits et le tableau 2 en présente l'analyse chiffrée.

Figure 4 : Hydrogrammes durant les périodes d'étiage, pour la Vaige, le Rhonne et l'Uzure Hydrograms during rates of flow at their lowest for the Uzure, the Vaige and the Rhonne 


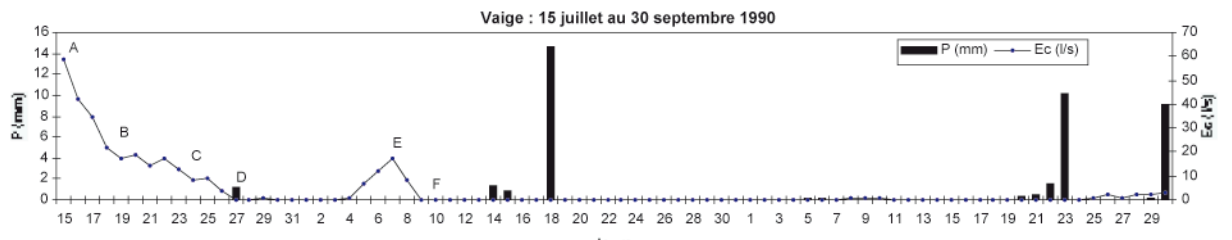
jours
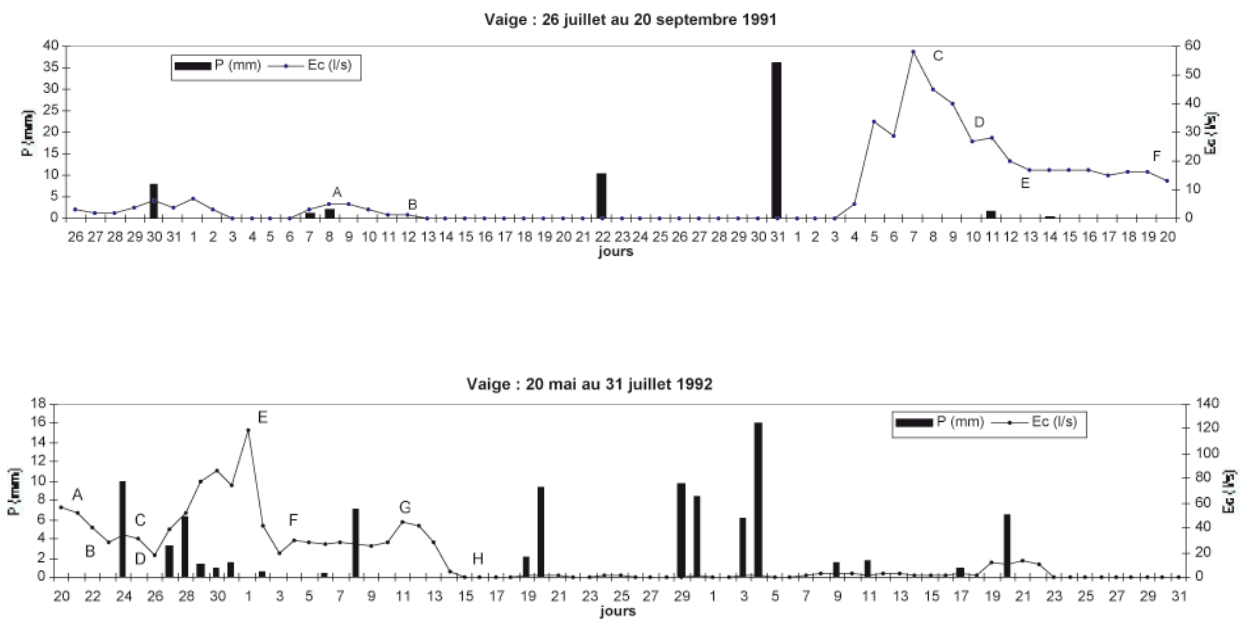

$4 b$
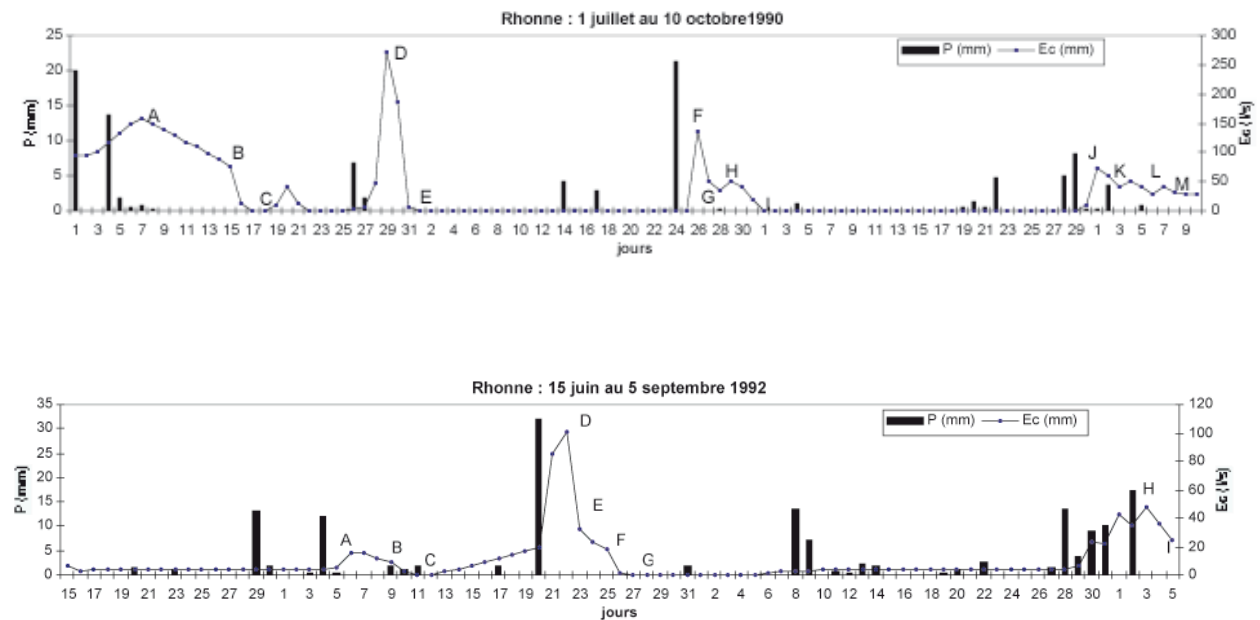


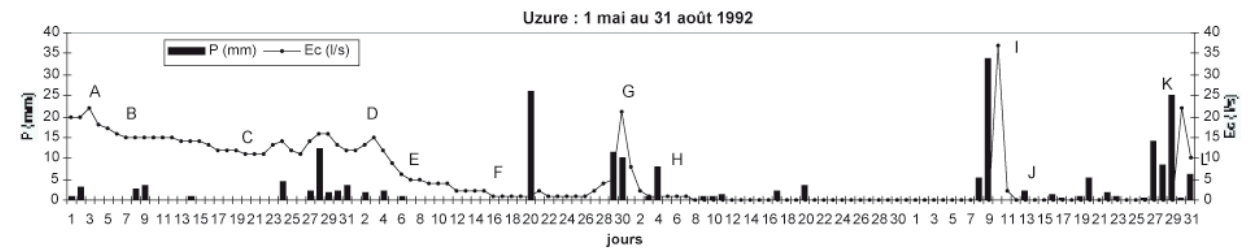

Tableau 2 : Les coefficients de tarissement et leurs interprétations Drying up coefficients and their explanations

\begin{tabular}{|c|c|c|c|c|}
\hline date & bassin versant & vecteur & $\begin{array}{l}\text { coefficient } \\
\text { de tarissement }\end{array}$ & mode d'écoulement \\
\hline \multirow[t]{2}{*}{$\begin{array}{l}10-11 \text { août } 1992 \\
25-26 \text { juillet } 1992 \\
29-31 \text { juillet } 1990 \\
29 \text { août- } 1 \text { er septembre } 1990 \\
22-23 \text { juillet } 1992 \\
9-10 \text { juillet } 1992 \\
30 \text { juin-6 juillet } 1992 \\
5-15 \text { juillet } 1992 \\
1-3 \text { juin } 1992 \\
26-28 \text { août } 1990 \\
30-31 \text { août } 1992 \\
7-8 \text { août } 1990 \\
11-14 \text { juin } 1992 \\
15-16 \text { juillet } 1990 \\
7-10 \text { septembre } 1991 \\
9-12 \text { août } 1991 \\
\end{array}$} & \begin{tabular}{|l|} 
Uzure \\
Rhonne \\
Rhonne \\
Rhonne \\
Rhonne \\
Rhonne \\
Uzure \\
Rhonne \\
Vaige \\
Rhonne \\
Uzure \\
Vaige \\
Vaige \\
Rhonne \\
Vaige \\
Vaige \\
\end{tabular} & \begin{tabular}{|l}
$I$ \\
$F G$ \\
$D E$ \\
$H I$ \\
$D E$ \\
$B C$ \\
$G H$ \\
$A B$ \\
$A B$ \\
$E F$ \\
$F G$ \\
$K L$ \\
$E F$ \\
$G H$ \\
$B C$ \\
$C D$ \\
$A B$ \\
\end{tabular} & $\begin{array}{l}-2,91777 \\
-2,89037 \\
-1,99634 \\
-1,25052 \\
-1,14938 \\
-1,09861 \\
-1,05199 \\
-0,8956 \\
-0,8927 \\
-0,86457 \\
-0,78848 \\
-0,75905 \\
-0,68946 \\
-0,62389 \\
-0,6054 \\
-0,53788 \\
\end{array}$ & $\begin{array}{l}\text { ruissellement } \\
\text { direct }\end{array}$ \\
\hline & & $\begin{array}{l}\text { Moyenne } \\
\text { E.Type } \\
\text { C.V. } \\
\end{array}$ & $\begin{array}{l}-1,18825 \\
0,75389 \\
-0,63 \\
\end{array}$ & \\
\hline \multirow[t]{2}{*}{$\begin{array}{l}24-26 \text { mai } 1992 \\
\text { 3-5 septembre } 1992 \\
\text { 3-6 juin } 1992 \\
23-25 \text { juillet } 1992 \\
15-17 \text { juillet } 1990 \\
20-23 \text { mai } 1992 \\
\text { 3-4 mai } 1992 \\
22-26 \text { juillet } 1990 \\
\text { 6-9 juillet } 1992 \\
\text { 1-4 octobre } 1990 \\
11-13 \text { septembre } 1991 \\
\text { 6-16 juin } 1992 \\
\end{array}$} & \begin{tabular}{|l|} 
Vaige \\
Rhonne \\
Uzure \\
Rhonne \\
Vaige \\
Vaige \\
Uzure \\
Vaige \\
Rhonne \\
Rhonne \\
Vaige \\
Uzure \\
\end{tabular} & $\begin{array}{l}C D \\
H I \\
D E \\
E F \\
A B \\
A B \\
A B \\
C D \\
A B \\
J K \\
D E \\
E F \\
\end{array}$ & $\begin{array}{l}-0,33249 \\
-0,32616 \\
-0,30366 \\
-0,28768 \\
-0,26109 \\
-0,23949 \\
-0,20067 \\
-0,19831 \\
-0,19492 \\
-0,17795 \\
-0,16252 \\
-0,15866 \\
\end{array}$ & $\begin{array}{l}\text { écoulement } \\
\text { hypodermique } \\
\text { (ressuyage } \\
\text { des sols) }\end{array}$ \\
\hline & & $\begin{array}{l}\text { Moyenne } \\
\text { E. Type } \\
\text { C.V. }\end{array}$ & $\begin{array}{l}-0,19267 \\
0,06355 \\
-0,33\end{array}$ & \\
\hline \multirow[t]{2}{*}{$\begin{array}{l}\text { 6-9 octobre } 1990 \\
17-18 \text { juillet } 1990 \\
\text { 13-20 septembre } 1991 \\
4-23 \text { mai } 1992\end{array}$} & \begin{tabular}{|l|} 
Rhonne \\
Vaige \\
Vaige \\
Uzure \\
\end{tabular} & $\begin{array}{l}\mathrm{LM} \\
\mathrm{BC} \\
\mathrm{EF} \\
\mathrm{BC} \\
\end{array}$ & $\begin{array}{l}-0,10601 \\
-0,09161 \\
-0,02962 \\
-0,02486 \\
\end{array}$ & $\begin{array}{l}\text { tarissement } \\
\text { des nappes }\end{array}$ \\
\hline & & $\begin{array}{l}\text { Moyenne } \\
\text { E. Type } \\
\text { C.V. }\end{array}$ & $\begin{array}{l}-0,06303 \\
0,04178 \\
-0,66\end{array}$ & \\
\hline
\end{tabular}

\section{La méthode d'analyse}

Elle repose sur la décomposition géométrique des courbes de tarissement par l'application de la loi de Maillet (1905) : $Q=$ Qo.e-a.t. Le tarissement (Q), exprimé ici en $1 / \mathrm{s}$, est une fonction exponentielle inverse du temps $(\mathrm{t})$ dont le coefficient de régression (a), appelé coefficient de tarissement est une caractéristique de l'écoulement sur chaque bassin versant, et son calcul permet de définir le mode d'écoulement : tarissement des sources, circulation hypodermiqueet écoulement rapide de crue. Les données obtenues ont permis de traiter 12 tarissements différents sur la Vaige, 13 sur le Rhonne et 7 sur l'Uzure. Pour ce qui concerne les périodes communes des étés 1990 et 1992, ces tarissements sont afférents à des crues issues des mêmes passages pluvieux. La comparaison est donc possible, car la proximité des trois bassins subodore un comportement hydrologique voisin. Le tri des 32 coefficients fait apparaître trois familles qu'il est possible d'interpréter comme la décrue du ruissellement et le 
tarissement des deux principaux réservoirs d'un bassin versant: le sol et le sous-sol. Les coefficients a, dont les valeurs sont comprises entre $-2,9177$ et $-0,53788$, traduisent un recul très rapide des débits : c'est la fin du ruissellement superficiel. Cet écoulement est directement conditionné par le volume des pluies, et l'échantillonnage des valeurs peut être très large, ce que montre un coefficient de variation de 0,66 . Le deuxième ensemble, dont la valeur est comprise entre $-0,33249$ et $-0,15866$, est constitué de coefficients plus faibles (moyenne $=-0,19267$ ) et plus homogènes (coefficient de variation de 0,36 ). La décrue est moins rapide, c'est l'écoulement hypodermique ou ressuyage des sols. Enfin, quelques rares exemples concernent une régression lente des débits (a compris entre -0,10601 et -0,02486) due au tarissement d'au moins deux types de réservoirs: une nappe phréatique au tarissement très lent ( $\mathbf{a}$ voisin de $-0,02)$ et probablement une nappe superficielle, souvent difficile à dissocier du ressuyage des sols, au tarissement plus rapide $(\mathbf{a}=-0,098)$.

\section{Les courbes de décrue et de tarissement}

Il faut rechercher des phases de tarissement regroupant successivement la décrue du ruissellement, le ressuyage des sols et le tarissement d'une nappe. Sur la Vaige, dont le bassin versant draine $233 \mathrm{~km}^{2}$, l'occurrence d'une averse de $37 \mathrm{~mm}$ le 31 août 1991 provoqua une onde de crue dont la pointe se situa en C le 7 septembre. Par la suite, en l'absence de nouvelles pluies importantes, au moins trois modes d'écoulement se sont succédé : du ruissellement entre $C$ et $D$, car le coefficient est élevé $(\mathbf{a}=-0,60540)$, le ressuyage du sol entre $\mathrm{D}$ et $\mathrm{E}(\mathbf{a}=-0,16.252)$ et les tarissements d'une nappe phréatique : $\mathbf{a}=-0,02.962$, car le coefficient est faible. On peut estimer avoir une vision correcte du fonctionnement de la Vaige. Toutefois l'hydrogramme CDEF n'est régulier que pour ces deux premiers membres $\mathrm{AD}$ et $\mathrm{DE}$, et le tarissement $\mathrm{EF}$ n'est qu'une suite de paliers, dont la tendance évoque la vidange d'une nappe souterraine. Il faut peut-être tenir compte du manque de fiabilité des mesures des très faibles débits.

Le Rhonne, petit bassin de $77 \mathrm{~km}^{2}$ offre le même type de réponse. Il n'existe pas de courbe de tarissement complète, mais il est possible de reconstituer le fonctionnement des différents réservoirs naturels du bassin versant. La décrue du ruissellement se trouve transcrite par les vecteurs FG : -0,66457 (août 1990) ou DE $=-1,44938$ (septembre 1992), qui appartiennent à des hydrogrammes afférents à de fortes averses : $21,5 \mathrm{~mm}$ le 26 août 1990 et $53 \mathrm{~mm}$ le 21 juillet 1992. Quant à l'hydrogramme JKLMN d'octobre 1990, il traduit la vidange naturelle des autres réservoirs du bassin versant: le ressuyage des sols (vecteurs $\mathrm{JK}=-0,17795$ et $\mathrm{LM}=-0,14384$ ) est suivi d'un écoulement beaucoup plus lent résultant probablement du tarissement des sources.

11 On parvient aussi à reconstruire une courbe de tarissement de l'Uzure $\left(21 \mathrm{~km}^{2}\right)$. Au début de mai 1992, la décrue $A B=-0,20067$ n'est qu'un ressuyage des sols, mais elle se prolonge par un long tarissement de 19 jours qui se réalise par paliers $(B C=-0,02466)$. La crue suivante $C D E$ ne concerne toujours que de l'écoulement hypodermique; en revanche, la crue $\mathrm{FGH}$, afférente à l'averse de $26 \mathrm{~mm}$ du 20 juin, provoqua un bref ruissellement dont la décrue $\mathrm{GH}=-1,05199$ fut rapide. 


\section{La place des assecs dans le fonctionnement des bassins}

12 L'influence des précipitations sur les débits n'est pas toujours aisée à interpréter car le grand espacement des pluviographes ne permet pas de mesurer précisément le volume d'eau réel tombé dans le bassin versant. Par exemple, en 1990, les 1,8 mm reçus par le bassin versant de la Vaige le 27 juillet semblent insuffisants pour expliquer la reprise de l'écoulement le 29 juillet, puis la pointe de $17 \mathrm{l} / \mathrm{s}$ entre le 7 et le 8 août, d'autant que les $17 \mathrm{~mm}$ de précipitations tombées du 14 au 18 août n'engendrent aucun écoulement.

Logiquement, l'assèchement naturel de l'écoulement devrait se traduire par les tarissements successifs de tous les réservoirs: sols, nappes superficielles, nappes souterraines. Or, cette évolution, analysée en 1976 et en 1989 sur l'Envigne, affluent de la Vienne (Giret, 1996 et 1998), ne s'observe pas ici.

Les assecs de la Vaige (fig. 4a) sont toujours précédés de tarissements rapides, mais les débits journaliers forment des séries décroissantes cohérentes : CD et EF en 1990, AB en 1991, CD et GE en 1992. En 1990, deux tarissements sont dignes d'intérêt : ABCD et EF. Le premier se produit après une crue survenue en juin et dont $A B C D$ constitue la phase de tarissement. Le coefficient de tarissement du vecteur $A B=-0,26.109$ traduit le ressuyage des sols, tandis que $\mathrm{BC}=-0,09.161$ évoque la vidange d'une nappe superficielle. À l'inverse, la pente anormalement élevée du vecteur $C D=-0,19.831$ ne correspond pas à la vidange d'une nappe. Or ce n'est pas une exception. Si le coefficient de décrue de $E F=-0,75.985$, traduit bien la fin d'une phase de ruissellement, la vidange $\mathrm{AB}=-0,53.788$, qui précède le long assec d'août 1991, n'évoque nullement celle d'une nappe ou d'un sol, mais bien une autre phase de ruissellement.

Le début de l'été 1992 fournit un élément de réponse. Des pluies abondantes $(20 \mathrm{~mm}$ entre le 20 mai et le 8 juin) ont engendré quatre crues qui culminèrent en $\mathrm{A}$ (20 mai), $\mathrm{C}$ (24 mai), E (1 ${ }^{\text {er }}$ juin) et $\mathrm{G}$ (11 juin). L'eau ruissela, comme le laisse à croire des coefficients élevés : $A B=-0,23.949, C D=-0,33.249, E F=-0,89170$ et $\mathrm{GH}=-0,68.946$. Puis ce fut l'assec, bien que les pluies n'aient pas cessé. Comme le suggère Lambert (1994), ces crues estivales (peu volumineuses en fait) pourraient résulter de l'eau de ruissellement sur le réseau routier et dans les fossés. Des averses brèves et violentes peuvent ruisseler sans recharger les nappes et, dans ce contexte, on passe directement du ruissellement à l'assec.

16 Toutefois, ni les $15 \mathrm{~mm}$ le 18 août 1990, ni les $11 \mathrm{~mm}$ le 23 août 1991 n'ont généré aucun écoulement. Que dire alors de l'absence d'écoulement en juin-juillet 1992, alors que 52 $\mathrm{mm}$ d'eau ont chu entre le 19 juin et le 3 juillet, dont $16 \mathrm{~mm}$ le 3 juillet. Il est vrai que Lambert (1994) et Gonot (1999) y verraient la résultante des prélèvements de la ripisylve, et, à l'inverse, la lente augmentation des débits de la Vaige en octobre 1990, pourrait s'expliquer par la réduction de ces prélèvements. Par exemple, Lambert (1994) a montré qu'un kilomètre linéaire de ripisylve consomme $0,73 \mathrm{l} / \mathrm{s} / \mathrm{jour}$ en juin, mais seulement 0,30 1/s/jour en octobre. En l'absence de précipitations, les oscillations brutales de débit sont interprétées différemment selon les auteurs : Callède (1977) pense qu'elles résultent davantage d'un rééquilibrage de la tension de l'eau dans le sol que des effets de l'évapotranspiration, alors que Lambert (1994) et Gonot (1999) y voient la résultante des prélèvements de la ripisylve.

17 Les assecs du Rhonne (fig. 4b) évoquent la probabilité de pompages agricoles. La crue qui culmina en A le 7 juillet 1990 conduit à un probable ressuyage des sols $A B=-0,08956$; mais, brutalement la vitesse de tarissement s'accélère : $B C=-0,62389$ 
vers un assec effectif le 17 juillet. La fin de ce mois connut de nouvelles pluies génératrices d'une crue; mais son tarissement $D E=-1,99634$ ne concerne que du ruissellement. Ce processus se reproduisit deux autres fois au cours de l'été 1990. Le tarissement FGHI, du 26 août au $1^{\text {er }}$ septembre achève une crue afférente aux $22 \mathrm{~mm}$ de pluies chus les 23 et 24 août. La décrue du ruissellement $F G=-0,55457$ fut interrompue par une averse de $1,5 \mathrm{~mm}$ (on notera la sensibilité de la structure hydrologique du bassin versant), mais sans autre apport pluvial la décrue reprit : HI =-1,25052.

Des événements semblables se sont reproduits au cours de l'été 1992. Il est tombé 24 $\mathrm{mm}$ de pluie entre le 29 juin et le 12 juillet, dont $12 \mathrm{~mm}$ le 29 juin. Cette eau circula dans les horizons du sol, ce que suggère le coefficient de décrue $A B=-0,19493$; puis il fait place à une vidange rapide $\mathrm{BC}=-1,09861$. Les $53 \mathrm{~mm}$ du 20 juillet ont engendré une nouvelle onde de crue qui se termine par un assec brutal le 26 juillet. Le vecteur $\mathrm{DE}=-1,44938$ traduit du ruissellement, le vecteur $\mathrm{EF}=-0,28768$ le ressuyage des sols, mais le tarissement des nappes qui devrait suivre est remplacé par une décrue rapide : $\mathrm{FG}=-2,89037$.

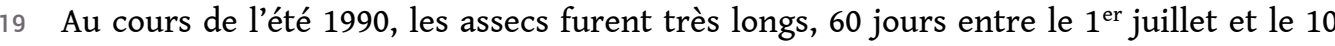
octobre, et tous furent précédés par une décrue rapide. L'écoulement souterrain par émergence des nappes phréatiques cessa, et les épisodes qui interrompirent les assecs ne relèvent que de l'écoulement rapide: écoulement hypodermique, voire ruissellement.

Quelles explications donner? La rapidité des tarissements évoque davantage les effets des pompages agricoles que le ruissellement sur chaussée. Le bassin versant a reçu 105 mm en 102 jours en 1990 et 175 mm en 93 jours en 1992 ; pourtant, du 15 juin au 5 juillet 1992, l'écoulement s'est stabilisé à 4 1/s. Plus qu'à une panne du limnigraphe, nous pensons que l'infiltration a été suffisante pour que les nappes émergent, et que malgré une succession d'épisodes pluvieux, l'écoulement se stabilise à l'amont du limnigraphe à cause des prélèvements pour l'irrigation.

21 L’Uzure (fig. $4 \mathrm{c})$, comme la plupart des très petits bassins versants $\left(21 \mathrm{~km}^{2}\right)$, réagit rapidement aux fluctuations pluviométriques à court terme : aussi, les différents modes de circulation sur et dans les sols et le sous-sol se succèdent sur une courte période. Du $1^{\text {er }}$ mai au 9 juillet 1992, la séquence commence par un fonctionnement normal des nappes : malgré de fortes averses ( $33 \mathrm{~mm}$ entre le 24 mai et le 6 juin, $26 \mathrm{~mm}$ le 26 juin, et $39 \mathrm{~mm}$ du 29 juin au 21 juillet) la tendance au recul général de l'écoulement pourrait n'être imputable qu'à l'évapotranspiration estivale. Mais l'assec survient le 9 juillet alors que des pluies n'ont pas cessé.

L'hydrogramme $A B C D E F$ représente une longue décrue interrompue, entre le 23 mai et le 3 juin, par quelques averses totalisant $30 \mathrm{~mm} . A B=-0,20067$ matérialise le ressuyage des sols, $B C=-0,02486$ le tarissement d'une nappe, $D E=-0,30366$, assez rapide, marque la reprise de l'écoulement hypodermique, mais $E F=-0,15866$ traduit un écoulement plus ambigu, car la valeur est à la limite du ressuyage d'un sol et du tarissement d'une nappe superficielle. L'analyse de la fin de la séquence apporte quelques indications. Les $26 \mathrm{~mm}$ de pluies chus le 20 juin sont restés sans effet, les $22 \mathrm{~mm}$ du 30 juin ont provoqué un ruissellement bref, mais violent: $\mathrm{GH}=-1,05199$. Malgré l'apport de quelques averses, juillet et août connurent l'assec à peine interrompu par la crue du 10-11 août $(\mathrm{IJ}=-2,9177)$ afférente à une averse de $39 \mathrm{~mm}$. Pourtant la lame écoulée au cours de ces 48 heures ne fut que de... $3,5 \mathrm{~mm}$ ou $10 \%$ de l'averse, le solde ayant satisfait l'évapotranspiration. D'ailleurs, dès le mois de mai, l'infiltration cessa au profit 
de cette dernière comme dans tout régime pluvio-évaporal. Il est donc logique que les nappes superficielles se soient taries, puis, que les nappes profondes aient cessé d'émerger. On peut alors interpréter l'ambiguïté du tarissement $E F=-0,15866$ qui marque la fin de l'émergence des nappes phréatiques : la vitesse anormale de la vidange témoigne d'une tension superficielle croissante au sein de l'aquifère, liée à l'impact des pompages qui, en rabattant la nappe, réduisent le débit.

En définitive, ces bassins ont tous connu des phases d'assèchement certaines. Ils ont en commun des coefficients de tarissement proche de $-0,20$, soit nettement supérieurs à ceux obtenus sur les cours d'eau plus pondérés ne s'asséchant pas, comme l'Huisne ou le Narais $(\mathbf{a}=-0,03)$. Le tarissement conduit à l'assec, mais le débit-seuil varie selon les bassins versants : très bas sur l'Uzure (1 à $2 \mathrm{l} / \mathrm{s}$ ), il monte pour la Vaige entre 3 et $5 \mathrm{l} / \mathrm{s}$ pour atteindre en moyenne $13 \mathrm{l} / \mathrm{s}$ sur le Rhonne. Ces variations s'expliquent par la différence de superficie des bassins, mais surtout par leur structure géologique : celui qui s'assèche avec un débit encore élevé, le Rhonne, traverse des terrains sédimentaires. Notre étude suggère qu'entre les phases d'assèchement, les brèves reprises d'écoulement sont dues, soit à des averses intenses entraînant sans doute une reprise du fonctionnement hydrologique, soit à un ruissellement sur des aires imperméabilisées, ou encore aux arrêts momentanés de l'irrigation.

\section{Les causes de l'assèchement}

\section{L'indigence des précipitations}

Les années de tarissement se caractérisent par un volume de précipitations inférieur à la moyenne, mais également par des rythmes saisonniers perturbés (Gravier et Larue, 1992). De 1988 à 1992, le déficit pluviométrique hivernal est manifestement lié à la modification des perturbations d'ouest et à la faiblesse de l'activité convective sur la région. Toutefois, toutes les années où la pluviométrie est inférieure à la moyenne n'engendrent pas forcément des phases d'assèchement : exemple l'année 1985.

Ainsi au Mans, les déficits pluviométriques annuels par rapport à la normale restent modestes avec $-15 \%$ en $1989,-12 \%$ en $1990,-23 \%$ en 1991 et $-7 \%$ en 1992 , et nettement inférieurs à celui de 1976 (-32\%). Par contre, les variations mensuelles s'avèrent être plus importantes (tableau 3). Après avoir connu une fin de saison froide plus humide que la moyenne, l'année 1989 est caractérisée par des déficits sévères et prolongés de mai à novembre. L'année 1990 est marquée par l'alternance de mois excédentaires, comme février, avril, juillet et octobre, et de mois très déficitaires, comme mars, mai, septembre et novembre. L'année 1991 subit un dérèglement assez semblable, mais les mois concernés ne sont pas tous les mêmes : si mai et septembre restent nettement déficitaires, mars et novembre deviennent excédentaires alors que février et décembre sont déficitaires. En 1992, seul le premier semestre enregistre des précipitations inférieures à la moyenne, juillet, août, novembre et décembre étant très largement excédentaires. Au total, durant les quatre années hydrologiques (août 1988-septembre 1992), les déficits occupent $70 \%$ du temps et l'hiver 1991-1992 se révèle le plus sec. 
Tableau 3 : Précipitations au Mans en 1976, 1988, 1989, 1990, 1991, 1992 et moyenne 1971-1988 Les chiffres en italique indiquent les mois déficitaires Rainfalls in Le Mans in 1976, 1988, 1989, 1990, 1991, 1992 and average 1971-1988 The months showing a deficit are in italic

\begin{tabular}{|l|r|r|r|r|r|r|r|r|r|r|r|r|r|}
\hline Le Mans & J & F & M & A & M & J & J & A & S & O & N & D & Année \\
\hline moy 1951-1980 & 64 & 61 & 55 & 43 & 55 & 56 & 44 & 54 & 57 & 53 & 71 & 67 & 679 \\
1976 & 26 & 48 & 29 & 7 & 12 & 0 & 48 & 6 & 85 & 80 & 73 & 51 & 465 \\
1988 & 119 & 86 & 71 & 56 & 70 & 20 & 80 & 15 & 40 & 39 & 51 & 31 & 678 \\
1989 & 30 & 72 & 102 & 98 & 30 & 28 & 29 & 6 & 13 & 30 & 38 & 104 & 580 \\
1990 & 48 & 91 & 6 & 52 & 33 & 51 & 46 & 35 & 28 & 100 & 44 & 63 & 597 \\
1991 & 103 & 24 & 67 & 44 & 17 & 50 & 29 & 11 & 34 & 53 & 74 & 13 & 519 \\
1992 & 8 & 33 & 51 & 35 & 46 & 21 & 57 & 95 & 50 & 44 & 111 & 76 & 627 \\
\hline
\end{tabular}

À l'échelle mensuelle, il existe un décalage entre les précipitations et l'écoulement (fig. 5) car, comme le souligne Grésillon (1996), les pluies doivent d'abord remplir le réservoir du sol que l'évapotranspiration a asséchée pendant la saison estivale. Pour ces cours d'eau à régime pluvio-évaporal, l'alimentation efficace se tient en saison froide quand l'évapotranspiration réelle est minimum, du fait de la faiblesse des températures et de la modestie de la demande d'une végétation en repos. En temps normal, la reconstitution des réserves du sol et des nappes souterraines est suffisante pour maintenir la pérennité de l'écoulement. Or de 1989 à 1992, l'infiltration insuffisante pendant la saison froide conduit aux périodes d'assèchement décrites. En outre, on peut noter une diminution progressive de l'apport pluvial de saison froide depuis l'automnehiver 1987-1988 jusqu'à la saison froide 1991-1992. Ainsi, pour le bassin versant du Rhonne, cet apport régresse-t-il de $588 \mathrm{~mm}$ à $240 \mathrm{~mm}$ en passant successivement par $347 \mathrm{~mm}, 340 \mathrm{~mm}$ et $425 \mathrm{~mm}$.

Figure 5 : Précipitations et écoulement dans le bassin de la Vaige de 1989 à 1992 Rainfalls and flow in the Vaige basin from 1989 to 1992

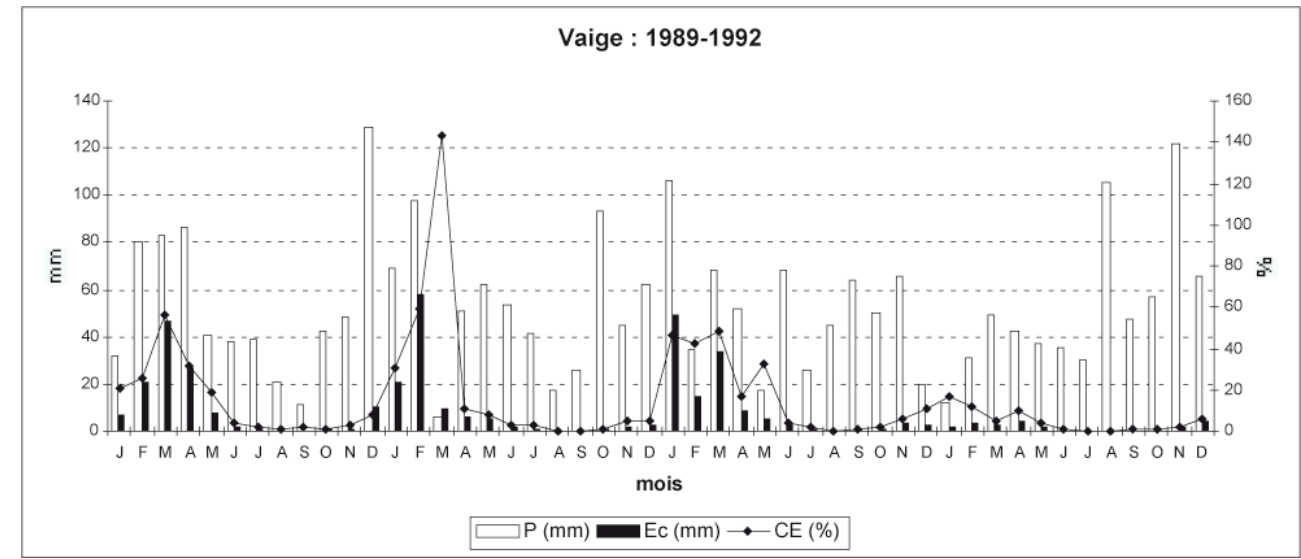

Ec : écoulement en $\mathrm{mm} ; \mathrm{P}$ : précipitations en $\mathrm{mm} ; \mathrm{CE}$ : coefficient d'écoulement en \% EC: flow in $\mathrm{mm}$; $P$ : rainfall in $\mathrm{mm}$; CE: discharge quotient in \%

Si les précipitations totales tombées entre 1989 et 1992 varient peu selon les bassins étudiés (entre $2290 \mathrm{~mm}$ pour le Chéran et $2527 \mathrm{~mm}$ pour la Vaige, durant les quatre années étudiées), l'écoulement et l'infiltration révèlent des différences plus importantes dans l'espace. Ainsi, le Rhonne écoule $241 \mathrm{~mm}$, soit un coefficient d'écoulement de $10 \%$, la Vaige, $374 \mathrm{~mm}$ et $14,8 \%$, l'Oudon, $311 \mathrm{~mm}$ et 12,6\%, l'Uzure, $391 \mathrm{~mm}$ et $16,6 \%$, et le Chéran, $483 \mathrm{~mm}$ et $21 \%$. Sur le socle, ce sont les plus petits bassins qui engendrent les plus forts coefficients d'écoulement. L'évolution des coefficients d'écoulement est peu différente d'un bassin à l'autre (fig. 6). L'infiltration (I 
$\mathrm{mm})$, qui assure la recharge de la réserve utile des sols et l'alimentation des nappes souterraines, est plus difficile à évaluer : $(\mathrm{I} \mathrm{mm})=$ précipitations $(\mathrm{P} \mathrm{mm})-$ [lame d'eau écoulée (Q $\mathrm{mm})+$ évapotranspiration réelle $(\mathrm{E} \mathrm{mm})]$. En utilisant pour $\mathrm{E} \mathrm{mm}$ les chiffres fournis par la Météorologie Nationale, on aboutit pour l'infiltration à des valeurs allant de $740 \mathrm{~mm}$ pour le Chéran à $1001 \mathrm{~mm}$ pour le Rhonne et $1042 \mathrm{~mm}$ pour l'Oudon.

Figure 6 : Évolution des coefficients d'écoulement de 1989 à 1992 Evolution of the discharge quotients from 1989 to 1992
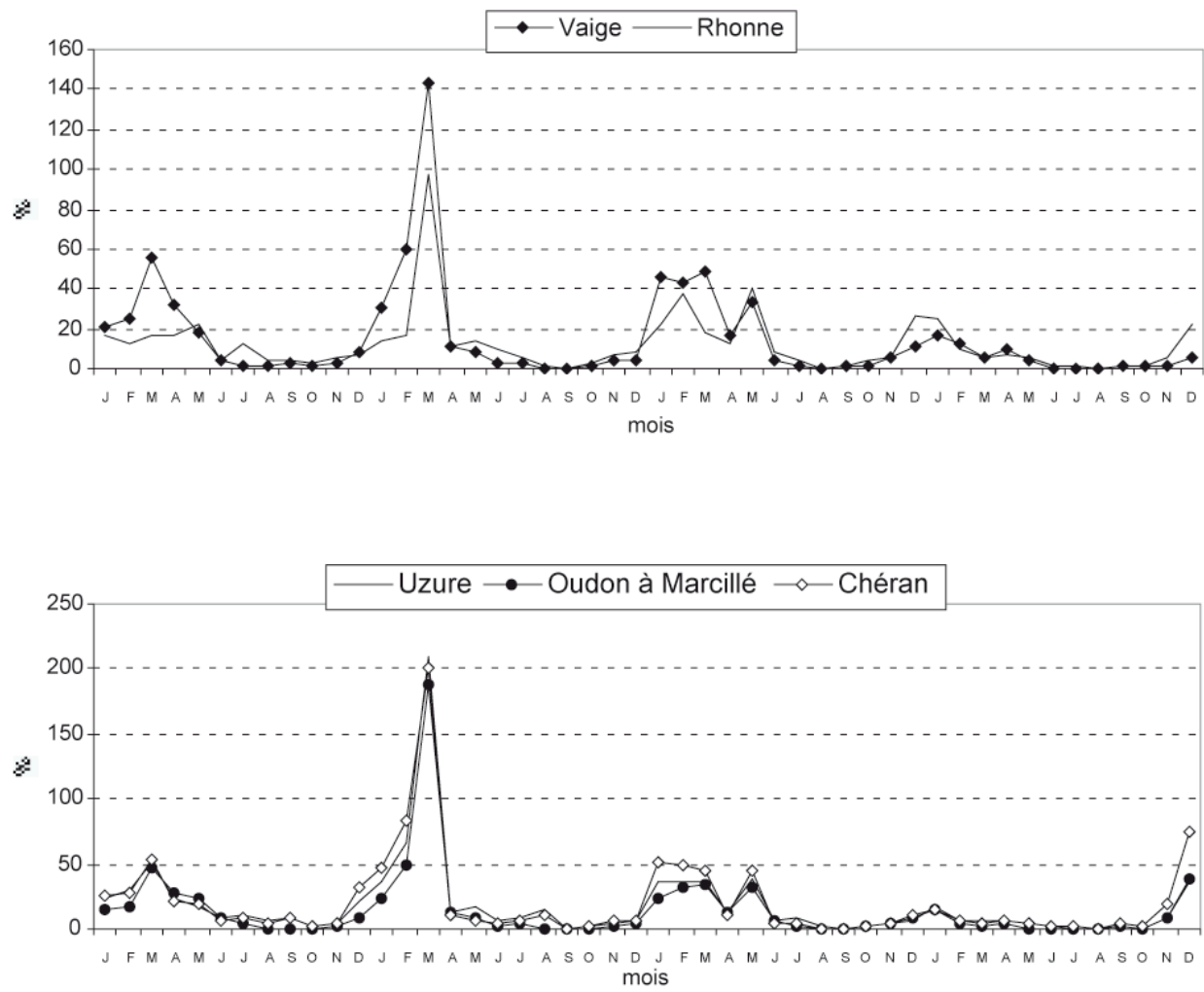

\section{Le rôle de la géologie et des formations superficielles}

En étudiant les bilans hydrologiques durant les années sèches, dans le cadre de petits bassins versants de la Bretagne occidentale, Mounier et al. (1993) ont mis en évidence des différences spatiales dues au climat, mais surtout à la géologie. Dans les régions granitiques, les réserves souterraines, conservées dans les arènes, atténuent les étiages, alors que dans les bassins schisteux, pratiquement dépourvus de réserves, l'arrêt de l'écoulement s'est produit en 1976 et en 1989.

Dans le bassin de la Maine, Larue (1994), en analysant les types de réponse des cours d'eau aux années de sécheresse 1989-1991, souligne aussi le rôle prépondérant de la structure et des sols dans l'explication des déficits hydrologiques. Les différences d'altitude, modestes à l'échelle de ce bassin qui est composé essentiellement de plateaux ne dépassant guère $200 \mathrm{~m}$ (les collines de Normandie culminent à $417 \mathrm{~m}$ et la confluence des trois rivières principales se situe à $14 \mathrm{~m}$ d'altitude), influent davantage sur la répartition spatiale des hauteurs de précipitations que sur les conditions d'écoulement liées au facteur pente. La morphométrie des bassins versants, en 
particulier les coefficients de compacité, joue un rôle plus important dans le développement des crues que dans celui des étiages.

Dans le socle du Massif armoricain, l'Oudon et la majeure partie de ses affluents (fig. 1) coulent sur des schistes briovériens (Herrouin et Rabu, 1990) dépourvus d'aquifères importants et développant des sols minces et argileux qui, lors des pluies, reconstituent d'abord leur réserve utile (100 mm environ) avant de permettre un écoulement. Ainsi, l'Uzure coule entièrement sur les schistes briovériens, le Chéran traverse des terrains d'âges plus variés (Précambrien, Ordovicien, Silurien) mais sur le plan lithologique, les schistes l'emportent largement sur les grès. Il en est de même des bassins versants localisés dans les synclinaux primaires de Laval et d'Angers, constitués de grès, de schistes et de calcaires..., et ne renfermant que des nappes souterraines morcelées et très peu importantes, incapables de soutenir les étiages prononcés. C'est le cas du bassin versant de la Vaige qui, malgré sa grande variété lithologique (schistes, conglomérats, grés, calcaires, houilles, roches volcaniques acides du Primaire, argiles, sables et graviers pliocènes), ne dispose que de réserves réduites. En effet, les calcaires carbonifères, qui sont fracturés, irrégulièrement karstifiés et le plus souvent constitués de bancs décimétriques séparés par des interlits schisteux (Ménillet et al., 1988), renferment un aquifère très morcelé qui fournit, par pompage, des débits très variables dans le temps. Les dépôts pliocènes ne forment que des placages résiduels plus riches en argile qu'en sable, ce qui n'est guère propice à la conservation de nappes importantes.

Dans la couverture sédimentaire, l'assèchement du Rhonne, qui s'effectue sur environ 2 $\mathrm{km}$ dans la partie aval du cours (Péan, 1994), s'explique à la fois par la lithologie et par la structure géologique de son bassin versant. Cet affluent de la Sarthe traverse la boutonnière du Bélinois d'est en ouest et coule, du fait du bombement anticlinal nordsud, principalement sur les matériaux marneux et argileux plutôt imperméables du Jurassique et du Crétacé inférieur. Toutefois, son cours amont recoupe des strates plus récentes, essentiellement sableuses et propices à l'alimentation d'aquifères. Cependant, en raison d'un pendage marqué vers l'ESE, ces derniers soutiennent surtout les débits des cours d'eau voisins qui s'écoulent dans le même sens, à savoir l'Aune et la Veuve. Le bassin hydrologique, plus petit que le bassin hydrographique, favorise la formation des étiages. Peu importantes, les formations superficielles sont de piètres réservoirs. Les sables éoliens qui couronnent les interfluves n'excèdent pas $1 \mathrm{~m}$ d'épaisseur; quant au remblaiement de fond de vallée qui ne dépasse pas $3 \mathrm{~m}$ de puissance, il est constitué de matériaux peu perméables. Les forages réalisés pour la construction de l'autoroute A28 révèlent la coupe type suivante, décrite du sommet vers la base: $0,20 \mathrm{~m}$ de terre végétale tourbeuse, $1,10 \mathrm{~m}$ d'argile sableuse, $0,70 \mathrm{~m}$ d'argile compacte orangée et bleue puis $1 \mathrm{~m}$ de sables argileux gris-vert, l'ensemble ravinant les argiles bleu-foncé du substrat secondaire.

\section{L'impact des activités anthropiques}

32 Si dans les bassins versants étudiés, l'assèchement apparaît inéluctable en cas de sécheresses prolongées, il est toutefois accéléré par certaines activités humaines.

Les prélèvements comptabilisés par l'agence de bassin Loire-Bretagne s'élèvent à 78,8

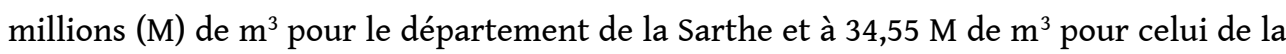
Mayenne en 1990, soit une croissance de 6 à $12 \%$ par rapport à 1988. Les prélèvements 
d'eau pour l'irrigation ont été multipliés par trois de 1970 à 1990 dans les départements de la Sarthe et de la Mayenne. Toutefois, toute l'eau prélevée n'est pas consommée, une partie étant rejetée dans la nature : la consommation réelle ne dépasse pas $15,5 \mathrm{M} \mathrm{de}^{3}$ pour la Sarthe et 6,2 M de $\mathrm{m}^{3}$ pour la Mayenne en 1990. En revanche, l'essentiel des pompages est réalisé pendant la période d'étiage, fixée par l'agence de bassin du $1^{\mathrm{er}}$ juin au 30 novembre, ce qui ne va pas sans provoquer une certaine concurrence entre les différents consommateurs: agriculteurs, industriels, EDF, collectivités locales. Cette concurrence nécessite une gestion globale de l'eau, comme le montre Sormail (1989) dans la vallée de la Garonne. Si les eaux superficielles restent les plus utilisées (83,5\% en Mayenne, 79,1\% en Sarthe), les aquifères voient leur part grandir dans la partie sédimentaire du bassin de la Maine à cause de la multiplication des forages pour les cultures de maïs en plein développement. Ainsi, de 1982 à 1988, les superficies irriguées déclarées sont-elles passées de 79000 ha à plus de 127000 ha pour l'ensemble du bassin. Dans la région de Sablé-sur-Sarthe et dans le Bélinois, entre les deux recensements agricoles de 1979 et 1988, les prairies naturelles ont régressé de plus de $50 \%$ à moins de $35 \%$ de la SAU au profit des terres labourées. Cette évolution s'est accompagnée de l'essor des cultures de printemps (maïs-grain, maïs-ensilage et tournesol) qui consomment beaucoup d'eau pendant la saison estivale (environ 2000 $\mathrm{m}^{3}$ par hectare).

De 1989 à 1992, la consommation réelle des agriculteurs a représenté $37 \%$ de la consommation totale alors qu'elle n'atteignait que $22 \%$ en 1987 et 1988. Ces prélèvements d'eau, qu'ils soient effectués dans les lits mineurs, dans les nappes phréatiques associées ou dans les nappes souterraines libres, peuvent déclencher le tarissement brutal des petits cours d'eau, comme celui des Deux Fonts, analysé par Breux (1993). Situé à l'est de Sablé-sur-Sarthe, au contact du socle et de la couverture sédimentaire, le ruisseau s'est asséché à plusieurs reprises et le rabattement de la surface piézométrique, causé par les pompages pour l'alimentation en eau potable et pour l'irrigation (800000 $\mathrm{m}^{3}$ par an), a entrâné l'infiltration de l'eau du lit vers l'aquifère. Ces fluctuations brutales sont probablement accentuées par la structure faillée et ondulée des strates sédimentaires, qui morcelle les nappes souterraines. Les reprises d'écoulement, qui surviennent sans qu'aucune précipitation ne soit tombée, comme pour la Vaige, du 3 au 9 juillet et du 7 au 11 septembre (fig. 4a), pourraient être dues à des arrêts momentanés de pompage pour l'irrigation: les pompages ne sont jamais continus, ne serait-ce qu'en raison du déplacement nécessaire des installations. L'absence de piézomètres dans les bassins étudiés ne nous a pas permis de suivre l'évolution du niveau des nappes phréatiques durant les années de sécheresse. En revanche, le suivi piézométrique entre 1993 et 1994 des nappes aquifères libres du Bajobathonien de la région de Parcé-sur-Sarthe prouve que les prélèvements des irrigants engendrent bien une baisse plus rapide du niveau de la nappe et que l'arrêt de l'irrigation à la mi-septembre se traduit par une remontée légère mais brutale du niveau de la nappe (fig. 7). Cette brusque remontée peut fort bien redonner un écoulement à un cours d'eau asséché. 


\section{niveaux par rapport au sol}

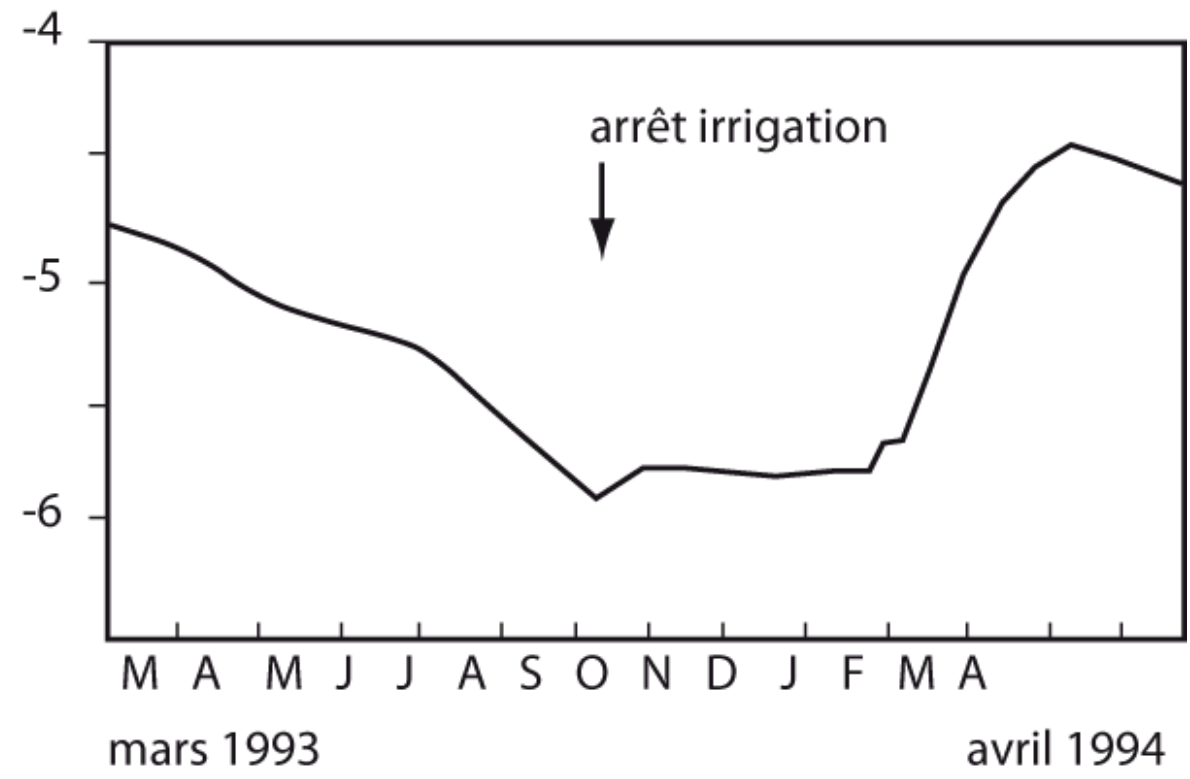

Source : Service Environnement Conseil Général de la Sarthe

L'utilisation du sol engendre des effets sur l'hydrologie (Bultot et al., 1990; Archer, 2000 ; Cosandey et Robinson, 2000). Les transformations rapides des paysages ruraux et des pratiques culturales (Dufour et al., 1990) favorisent le ruissellement direct aux dépens de l'infiltration, ce qui tend à accentuer la déjà faible pondération des écoulements. En effet, tous les bassins versants subissent la multiplication des surfaces imperméabilisées, le déboisement (les étendues boisées, forêts, taillis et haies, restent inférieures à $10 \%$ de l'espace), et le développement des labours aux dépens des prairies naturelles. Ainsi, au printemps, lorsque la couverture végétale est encore très faible et bien que le sol ne soit pas gorgé d'eau, les averses orageuses engendrent des crues brutales bien visibles sur les hydrogrammes (fig. 4). Ces pics ne peuvent s'expliquer que par un ruissellement de surface induit par la formation, sous l'impact des gouttes de pluie, d'organisations pelliculaires de surface (OPS) qui limitent considérablement l'infiltration (Boiffin, 1984). Ce ruissellement rapide fait que les nappes souterraines, mal alimentées pendant la saison froide, ne peuvent soutenir les étiages estivaux.

La prolifération de petits plans d'eau dans la plupart des lits majeurs, comme celui du Rhonne, stimule l'évaporation qui peut atteindre entre 0,5 et $1 \mathrm{l} / \mathrm{s} / \mathrm{ha}$ en période estivale (Péan, 1994). Toutefois, leur faible importance en superficie (0,5\% du bassin versant) limite leur influence. Ces étangs n'ont par ailleurs qu'une faible utilité dans le soutien des étiages, contrairement aux barrages de retenue dont la construction est projetée sur le Rhonne afin d'éviter les tarissements à répétition. En revanche, les ripisylves, qui forment des liserés presque continus le long des rivières étudiées, contribuent à réduire fortement les débits de base lors de la période végétative (Gonot, 1999). Lors des périodes de forte insolation et de forte activité photosynthétique, la forte consommation d'eau par les ripisylves accélère l'assèchement. À l'opposé, à l'automne, la réduction de la photosynthèse limite les prélèvements de la ripisylve et favorise la reprise de l'écoulement et l'augmentation des débits de base. En outre, le non-entretien des berges conjugué à l'impact des dernières sécheresses, ont favorisé 
l'envahissement des lits par une végétation plus ou moins exubérante d'arbrisseaux (aulnes principalement), de ronces, de fougères, d'orties... qui consomment une partie de l'eau et freinent l'écoulement. Cette végétation accentue les tarissements, mais présente aussi localement un risque d'aggravation des crues, par les embâcles qu'elle peut produire.

\section{Conclusion}

Bien que rare dans le bassin de la Maine, l'assèchement du lit des cours d'eau s'est produit lors des années de sécheresse, comme 1976, 1990, 1991 et 1992, mais pas en 1989. Provoqué par l'indigence des précipitations, le tarissement survient lorsque l'écoulement de base n'est plus alimenté par les nappes souterraines. Cette situation résulte de conditions morphostructurales particulières, mais aussi du rythme et de l'intensité des prélèvements d'eau dans le bassin versant. L'analyse des arrêts et des reprises d'écoulement révèlent deux cas de figure :

- Les bassins versants pratiquement dépourvus de réserves souterraines, comme celui de l'Oudon sur le socle schisteux du Massif armoricain, ont des écoulements contrôlés par les précipitations. L'assèchement peut être précoce et les reprises d'écoulement sont dues à des averses suffisamment abondantes pour engendrer un ruissellement de surface et/ou un écoulement hypodermique.

- Les bassins qui possèdent des nappes souterraines discontinues pour des raisons structurales, comme la Vaige sur le socle, le Rhonne sur la couverture sédimentaire du Bassin parisien ou les Deux-Fonts à cheval sur les deux, subissent des phases d'assèchement entrecoupées de reprises d'écoulement qui ne sont pas toutes provoquées par des précipitations. Les pompages pour l'irrigation, irréguliers dans le temps, engendrent des fluctuations du niveau piézométrique qui, tantôt stoppent l'écoulement de base, tantôt le régénèrent. Enfin, les prélèvements inégaux dans le temps des ripisylves jouent aussi un rôle important dans les fluctuations des débits de base.

Entre 1989 et 1992, l'accentuation dans le temps de la durée des assèchements s'explique principalement par la faiblesse des pluies efficaces de saison froide qui n'a pas permis une recharge suffisante des nappes souterraines. Le bassin de l'Envigne en Poitou-Charentes, a enregistré une évolution identique (Giret, 1999). Les conséquences des assecs sur les biocénoses ne nous sont pas apparues catastrophiques, mais cela mériterait une étude spécifique.

L'analyse des hydrogrammes est donc une méthode efficace pour l'étude de l'assèchement des petits cours d'eau et la compréhension du fonctionnement hydrologique de petits bassins versants. Toutefois, elle nécessite d'être couplée à un suivi piézométrique des nappes qui peuvent soutenir les étiages. Cela favoriserait la prévision des assèchements (Michel, 1992) dans les régions agricoles utilisant l'irrigation. 


\section{BIBLIOGRAPHIE}

ARCHER (D. R.), 2000. - « Indices of flow variability and their use in assessing the impact of land use changes ", British Hydrological Society, Seventh National Symposium, p. 267-273.

BoIfFin (J.), 1984. - La dégradation structurale des couches superficielles du sol sous l'action des pluies, thèse de docteur-ingénieur, INAPG, Paris, 320 p. + annexes.

Breux (C.), 1993. - L'eau dans le bassin des Deux Fonts, mémoire de Maîtrise de géographie, Université du Mans, 72 p.

Bultot (F.), Dupriez (G. L.), Gellens (D.), 1990. - « Simulation of land use changes an impacts on the water balance : a case study for Belgium », Journal of Hydrology, n 114, p. 327-348.

BUTLER (S.S.), 1957. - Engineering Geology, Prentice Hall, 356 p.

CALLÈDE (J.), 1997. - Oscillations journalières du débit des rivières en l'absence de précipitations, thèse de docteur-ingénieur, Université de Paris VI, 131 p.

COSANDEY (C.), 1993. - Forêt et écoulements : rôle de la forêt sur la formation des crues et le bilan d'écoulement annuel. Impact d'une coupe forestière. Laboratoire de géographie physique P. Birot, Meudon, CNRS, $81 \mathrm{p}$.

-, 2003. - Les eaux courantes, Paris, Belin, 240 p.

Cosandey (C.), Robinson (M.), 2000. - Hydrologie continentale, Paris, Armand Colin, 360 p.

Dufour (J.), Gravier (J.), Larue (J.-P.), 1990. - « Fortes pluies et érosion des sols : l'orage de mai 1988 dans la Sarthe », Bulletin de l'Association de Géographes Français, n² 2, p. 159-170.

GIRET (A.), 1993. - «Fonctionnement hydrologique des altérites et des sols sur le glacis des Mauges (exemple du bassin de l'Hyrome en Maine-et-Loire) », Quaternaire, 4, n 2-3, p. 111-118.

-, 1996. - « Contribution à l'analyse des décrues et des tarissements dans la connaissance des réserves hydrologiques : l'exemple de l'Envigne entre 1968 et 1994 », Sécheresse, vol. 7, nº 4, p. 253-268.

-, 1998. - « Rôle du paysage naturel dans l'occurrence des crues : l'exemple de l'Envigne, un affluent poitevin de la Vienne », Poitiers, Norois, $n^{\circ} 179$, p. 543-566.

-, 1999. - « 1971-1976 et 1989-1992 : deux anomalies de l'écoulement en Poitou-Charentes. Exemple de l’Envigne », Sécheresse, vol. 10, n² 2, p. 105-115.

-, 2002. - «L'hydrologie de la Charente entre nature et anthropisation (1960-1994) », Sécheresse, vol. 13, n 2, 105-112.

Gonot (B.), 1999. - « L'incidence des prélèvements ripicoles sur le débit des cours d'eau du bassin de la Midouze ", Annales de Géographie, n 606, p. 191-199.

GRAVIER (J.), LARUE (J.-P.), 1992 - « La sécheresse de la fin des années 80 dans le bassin de la Maine : analyse climatique, impacts hydrologiques et écologiques », Norois, $\mathrm{n}^{\circ}$ 154, p. 163-184.

GRÉSILLON (J.-M.), 1996. - « Le bassin versant. Échelles d'analyse et compréhension des processus ", Géomorphologie, n 3, p. 3-16.

HERROUIN (Y.), RABU (D.), 1990. - Notice explicative de la feuille Châteaubriant à 1/50 000, Orléans, BRGM, $51 \mathrm{p}$. 
LAMBERT (R.), 1975. - Recherches hydrologiques sur le sud-est du bassin de la Garonne, thèse d'État, Université de Toulouse, 2 t., 750 p.

LAMBERT (R.), 1994. - « De la crue du débit de base des rivières du bassin garonnais d'août à octobre alors que perdure la sécheresse atmosphérique ", Dossier Revue de Géographie alpine, $\mathrm{n}^{\circ} 12$, p. 117-123.

LARUE (J.-P.), 1994. - « Les caractéristiques hydrologiques de trois années consécutives de sécheresse $(1989,1990$ et 1991) dans le bassin de la Maine ", Norois, n 163, p. 429-441.

MAILlET (E), 1905. - Essais d'hydraulique souterraine et fluviale, Paris, Herman éd., 218 p.

MÉnillet (F.), Plaine (J.), MANigault (B.), Le hÉRISsÉ (A.), 1988. - Notice explicative de la feuille Meslaydu-Maine à 1/50 000, Orléans, BRGM, $79 \mathrm{p}$.

MÉRILLON (Y.), 1992. - « Sécheresse, gestion de l'eau et pratiques agricoles », L'Information agricole, $\mathrm{n}^{\circ} 649$, p. $25-26$.

Michel (C.), 1992. - « Comment prévoir l'assèchement des cours d'eau ? " L'Information agricole, $\mathrm{n}^{\circ}$ 649 , p. 26-27.

Mounier (J.), DubReuil (V.), Le hÉNAFF (F.), 1993. - « Sécheresses et rivières en Bretagne Occidentale ", dans L'eau, la terre et les hommes, Mélanges en l'honneur de R. Frécaut, Nancy, Presses Universitaires de Nancy, p. 263-272.

PÉAN (F.), 1994. - Le bassin versant du Rhonne : un écoulement perturbé, mémoire de Maîtrise de géographie, Université du Mans, $144 \mathrm{p}$.

SORMAIL (L.), 1989. - «Besoins en eau et politique de soutien d'étiage dans la vallée de la Garonne ", Revue de Géographie des Pyrénées et du Sud-Ouest, n 4, p. 535-548.

\section{RÉSUMÉS}

L'étude des débits journaliers des cours d'eau du bassin de la Maine, gérés par la DIREN Pays de la Loire, montre que seuls cinq bassins versants (l'Oudon et ses tributaires, l'Uzure et le Chéran, la Vaige et le Rhonne) ont subi des périodes de tarissement en 1990, 1991 et 1992, mais pas en 1989. Soumis à des précipitations déficitaires par rapport à la moyenne mais relativement homogènes dans l'espace, les bassins enregistrent des durées d'assèchement assez différentes. La plus ou moins grande importance des réserves (sols, nappes superficielles et souterraines), qui dépend essentiellement des conditions morphostructurales et pédologiques, explique ces variations. Lorsqu'elles sont formées de schistes, les régions du socle du Massif armoricain apparaissent très sensibles aux risques de tarissement. Néanmoins, les prélèvements opérés par les ripisylves et l'impact des activités humaines, en particulier les prélèvements d'eau pour l'irrigation, sont à prendre en compte pour comprendre certaines fluctuations rapides de l'écoulement, comme par exemple celles du Rhonne sur la couverture sédimentaire du Bassin parisien.

An analysis of the hydrometric data concerning the streams of the Maine basin, which is managed by DIREN Pays de la Loire, shows that only five drainage basins (the Oudon and its tributaries, the Uzure and the Chéran, the Vaige and the Rhonne) suffered periods of drying up in 1990, 1991 and 1992, but not in 1989. With rainfall below average, but relatively homogeneous in space, the drainage basins have different drying up periods. More or less important groundwaters, depending essentially on morphostructural and soil conditions, account for these fluctuations. The schistous areas of the Massif armoricain are particularly liable to drying up. Therefore, water comsumption by the riparian vegetation and the impact of human activities, 
mainly water-consumption, must be taken into account in order to understand the rapid fluctuations of some streams, such as the Rhonne river on the sedimentary layers of the Paris basin.

INDEX

Mots-clés : agriculture, bassin versant, hydrogrammes, hydrologie, crue, tarissement Keywords : agriculture, drainage basins, drying up, flood, hydrology

Index géographique : France, Loire (fleuve et vallées), Maine (rivière et vallées), Pays de la Loire

\section{AUTEURS}

\section{JEAN-PIERRE LARUE}

Géodynamique des milieux naturels et de l'environnement, Université Paris 12 Val de Marne, 61 avenue du Général de Gaulle, 94010 Créteil cedex,

larue@univ-paris12.fr

\section{ALAIN GIRET}

UFR-LSHS, Université Paris 13, 99 avenue Jean-Baptiste Clément, 94430 Villetaneuse, agiret@wanadoo.fr 